\title{
SOBRE LAS CONCEPCIONES EN DINÁMICA ELEMENTAL DE LOS ADOLESCENTES FORMALES Y CONCRETOS Y EL CAMBIO METODOLÓGICO
}

\author{
ACEVEDO, J.A., BOLÍVAR, J.P., LÓPEZ-MOLINA, E.J. y TRUJILLO, M. \\ Seminario de Física y Química del I.B. Alonso Sánchez.
}

\section{SUMMARY}

This paper summarizes the main results of a research carried out with 15 to 18 year old students in the second year of Spanish Secondary Higher Education. Frequencies of wrong answers, based on the students' misconceptions about dynamics, and the Piagetian levels of concrete/formal proportional reasoning, are correlated. Conceptual change after a learning period is also analyzed. Short term results show that only students with higher formal reasoning levels improved in a significant way. Finally, considerations about methodology in class, in order to overcome pupils' difficulties in learning physics are also discussed.

\section{INTRODUCCIÓN}

¿Qué enseñar? En relación con esta pregunta, aplicada en nuestro caso a las ciencias experimentales, es bien conocida la dicotomía entre dar prioridad a los aspectos conceptuales o a los procesos propios de la actividad científica.

Los partidarios de dar una relevancia máxima a los procesos podrian ser interpretados como seguidores de las posiciones piagetianas más ortodoxas aplicadas al aula. Desde esta perspectiva, la enseñanza de las ciencias debe estar encaminada a facilitar el desarrollo del razonamiento formal de los estudiantes, el cual constituirá la herramienta necesaria y suficiente para poder acceder al conocimiento científico (Pozo y Carretero 1987). Sin embargo, desde la aparición de la obra clásica sobre el pensamiento formal de Inhelder y Piaget (1955), y sin dejar de reconocer su gran importancia, muchas investigaciones han falseado diversos aspectos de la misma. Los resultados muestran que la capacidad operatoria formal no constituye una estructura de conjunto, sino más bien diversas estrategias cognitivas apropiadas para la solución de problemas, las cuales no parecen adquirirse de forma unitaria, influyendo además el contenido de la tarea a resolver, lo que supone tener que distinguir entre competencia y actuación formal (Carretero 1980).

Desde otro punto de vista, se considera necesario que, sin renunciar a la formación procesual de los alumnos en la metodología científica, la enseñanza de las ciencias experimentales se oriente también hacia la adquisición de los esquemas conceptuales básicos de las mismas. En esta linea ha alcanzado gran importancia la corriente investigadora que, desde los trabajos pioneros de Viennot $(1979 \mathrm{a}, \mathrm{b})$, se orienta al estudio de la ciencia intuitiva de los escolares, empezando a ser numerosas las referencias bibliográficas sobre el tema (Carrascosa 1983, 1985, Furió 1986).

Estas ideas previas de los adolescentes se producen frecuentemente como consecuencia de la interacción cotidiana con el mundo que los rodea. Son resistentes al cambio, ya que ni siquiera el diseño de materiales específicos para la enseñanza de un concepto parece asegurar el abandono de la preconcepción correspondiente (Otero y Brincones 1987). Podrían constituir esquemas conceptuales alternativos ampliamente compartidos, lo que ha hecho sospechar de la existencia de ciertos mecanismos restrictivos, ligaduras cognitivas, en la forma que tenemos los humanos al procesar la información para elaborar ciertos constructos conceptuales (Keil 1981, Sebastiá 1987 a,b). Además, todo parece indicar que están organizados jerárquicamente, constituyendo teorias funcionales, útiles para la predicción y el control de los sucesos habituales.

Con la pretensión de investigar en el aula los aspectos anteriores, hemos diseñado un plan de trabajo en el que se consideren tanto las características cognitivas de los escolares, desde un punto de vista piagetiano, como sus ideas intuitivas en relación con la interpretación de fenómenos físicos familiares. Para ello hemos elegido, por una parte, la dinámica como área conceptual de la física clásica de gran relevancia en los currículos de ciencias, y, por otra, hemos tomado como referencia la competencia en el razonamiento proporcional, que 
consideramos uno de los esquemas operatorios más importantes para la interpretación cuantitativa de los datos de experiencias científicas.

\section{DEFINICIÓN DEL PROBLEMA, HIPÓTESIS Y VARIABLES}

El problema se ha establecido en forma de preguntas como las siguientes: ¿Existe inicialmente una diferen. cia significativa entre los adolescentes formales y con* cretos en la frecuencia de respuestas erróneas con respecto al esquema conceptual de la dinámica de Newton? ¿Son cualitativamente similares sus respuestas? ¿Hay diferencias después de un corto período de apren. dizaje en el aula, en torno a 15 sesiones, utilizando una metodologia supuestamente encaminada a favorecer el cambio conceptual? ¿Evolucionan positiva y significativamente los estudiantes con la ayuda de la metodología y de los materiales de aprendizaje utilizados?

En torno a las cuestiones anteriores hemos formulado la siguientes hipótesis:

(A) Puesto que los resultados de otros trabajos apuntan hacia una interpretación general espontánea o inn tuitiva, que es compartida con pequeñas variantes por la mayoría de los sujetos procedentes de diferentes medios o contextos y con edades diversas (Sebastiá 1984), creemos que no debe existir una diferencia significativa de partida entre los alumnos formales y concretos al responder a las cuestiones de dinámica elemental que les hemos planteado.

(B) Por el contrario, esperamos que aparezcan diferencias después del periodo de aprendizaje. Nuestra suposición se basa en los resultados obtenidos en algunas investigaciones, que muestran que los alumnos con competencia formal son los que más se beneftcian de un tratamiento metodológico que permita un aprendizaje alejado de una resolución superficial y acrítica de los problemas planteados haciendo uso de actividades de investigación: mediante el trabajo en grupo; esto es, que implique la interacción social entre iguales (Aguirre de Cárcer 1985).

(C) Estas mismas consideraciones nos hacen suponer que la evolución de los esquemas conceptuales en el sentido deseado será más probable, en un período corto, para aquellos alumnos con competencia formal, preferentemente consolidada, sobre todo teniendo en cuen. ta las caracteristicas del esquema conceptual newtoniano. Además, esta evolución positiva, aunque significativa, no debe ser muy acusada cuantitativamente. Esto último queda fundamentado si, como parece ser, los esquemas conceptuales alternativos son muy persistentes (Driver 1986) siendo posible que incluso coexistan con el nuevo esquema conceptual recién adquirido (Sebastiá $1987 \mathrm{a}, \mathrm{b})$.

Dos son las variables que hemos manejado en nuestra investigación la competencia formal/concreta en el ra- zonamiento proporcional, dicotomizada a partir de su medida obtenida utilizando dos tareas de proporcionalidad ya comentadas en otro lugar (Acevedo et al. $1987 \mathrm{a}, \mathrm{b})$, y la comprensión de conceptos de dinámica newtoniana elemental, evaluada con una prueba disc. nada especificamente para este trabajo, cuyas principales características se detallan más adelante.

Otros factores han sido controlados hasta lo razonable en condiciones ordinarias de aula. Asi se ha empleado la misma metodología didáctica, idénticos ma. teriales de aprendizaje, con igual ritmo de trabajo y un horario escolar similar, siendo el mismo profesor el que ha trabajado con los dos grupos naturales de alumnos que constituyeron la muestra investigada; además, am. bos grupos presentan una distribución muy similar en la competencia cognitiva medida.

\section{MUESTRA INVESTIGADA Y PROCEDIMIEN- TO DE AULA}

La muestra quedó constituida por 65 adolescentes (15-18 años, con edad media de 16.0 años) estudiantes de $2^{\circ}$ de BUP, pertenecientes a una población socioeconómica de clase media y media-baja, escolarizados en un Instituto de Bachillerato de Huelva capital, ubicado en una barriada urbana. Los niveles cognitivos de estos alumnos, con referencia al razonamiento proporcional, se muestran en la Tabla I, no variando significativamente de los obtenidos para una muestra mucho más ampliada de estudiantes del mismo curso de varios Institutos de Huelva capital y provincia.

Tabla 1

Clasificación piagetiana de la muestra investigada en función de la competencia formal/concreta en el razonamiento proporcional.

\begin{tabular}{|c|c|c|}
\hline EIVEZRS & $\begin{array}{c}\text { PRBCUIRECIA } \\
\text { NRSOLUTA }\end{array}$ & $\begin{array}{l}\text { PRECURESCIA } \\
\text { RETATIVA }(\cdots)\end{array}$ \\
\hline IIt B & 15 & 23.1 \\
\hline $131 \mathrm{~A}$ & 12 & 18. 5 \\
\hline $11 \mathrm{~B}$ & 28 & 43.1 \\
\hline II A & 10 & 13.4 \\
\hline
\end{tabular}

El procedimiento seguido en el aula puede resumirse en las siguientes estapas:

$1^{\text {a }}$. Una fase exploratoria de las ideas previas de los alumnos, obtenidas primero mediante la resolución individual de la prueba inicial de dinámica y cespués, al día siguiente, a partir de la realización de la misma en pequeños grupos de 4.65 alumnos. El análisis de las 
respuestas individuales permitió clasificar los tipos más frecuentes de éstas, que se confirmaron tras el estudio de las explicitadas por los grupos, ya que desaparecieron precisamente las más difícilmente clasificables en la prueba individual.

$2^{a}$. Un periodo de aprendizaje, de unas 15 horas, en el que los estudiantes realizaron diversas actividades extraídas y adaptadas de los programas-guía para la clase de física elaborados por el Grupo Recerca-Faraday (1985), que son una adaptación para la Enseñanza Media española del Harvard Project Physics, proyecto norteamericano para el aprendizaje de la física que ha sido comentado por Rutherford (1971) y Holton (1978) entre otros. Estos materiales están basados en una metodologia por descubrimiento guiado, así como en la evolución histórica de los conceptos como hilo conductor de la estructura de los contenidos. Tienen la finalidad de dar una visión humanística y evolutiva de la ciencia, que ponga de manifiesto las dificultades y los logros de la actividad científica.

$3^{\text {a }}$. Finalizado el período de aprendizaje anterior, los alumnos realizaron individualmente otra prueba similar a la inicial con el fin de comprobar la persistencia o el cambio de las respuestas recogidas, asi como de conocer la eficacia del método de aprendizaje y los materiales empleados, en el sentido de favorecer la evolución hacia la aplicación del esquema newtoniano.

\section{CARACTERÍSTICAS DEL INSTRUMENTO DE MEDIDA Y DISEÑO ESTADÍSTICO}

Las pruebas inicial y final de dinámica elemental implican dibujar, describir e interpretar las fuerzas que actúan sobre un cuerpo en movimiento en diversos casos, los cuales se desglosan en una o más cuestiones correspondientes a las distintas posiciones del movil. Cada cuestión constituye un item puntuable, pero el conjunto está destinado también a detectar cualitativamente, si es que existe, un esquema conceptual del sujeto que sea coherente con sus respuestas.

En este trabajo, ambas pruebas se han empleado con un formato abierto, ya que hay que completar los dibujos del movimiento del cuerpo con las fuerzas que intervienen, asi como dar una explicación breve en la que se describan o interpreten dichas fuerzas. Posteriormente, para otros trabajos que se encuentran en fase de elaboración, hemos modificado el formato utilizado presentando en cada cuestion cuatro opciones con las fuerzas ya dibujadas, seleccionadas de las respuestas más frecuentes encontradas en esta investigación, y añadiendo la posibilidad de una quinta opción libre en la que pueden dibujarse las fuerzas si ta respuesta del alumno no encaja con las que se le ofrece en cada ítem. No obstante, hemos mantenido la explicacion abierta. En el Anexo se muestra la prueba inicial en su versión actualizada.

La fiabilidad interna de las pruebas, desde el punto de vista de su consistencia interna, es alta. Asi, por ejem- plo, para la prueba inicial, los coeficientes de Guttman, Rulon y Sperman-Brown dan el mismo valor: $\mathrm{Rxx}=0.91$.

Por otra parte, dadas las características de las variables manejadas y del procedimiento que hemos seguido, el contraste estadístico de hipótesis se ha realizado mediante la prueba de Student para muestras independientes, en el caso de comparar las puntuaciones obtenidas en cada prueba con la competencia formal/concreta en el razonamiento proporcional; o para muestras dependientes, cuando se comparan las puntuaciones entre ambas pruebas referidas por separado a los alumnos formales o a los concretos. En este último caso también se ha considerado qué điferencia de puntuaciones es estadísticamente significativa con una probabilidad de, al menos, el $95 \%$. El nivel de significación que nos hemos matcado para validar estadísticamente nuestras hipótesis es, como mínimo, del $95 \%$ ( $\mathrm{p} \quad 0.05$ ). Finalmente, en aquellos casos en que la diferencia sea estadísticamente significativa se ha añadido un coeficiente de correlación biserial puntual $(\mathrm{rb}, \mathrm{p})$.

\section{ANÁLISIS E INTERPRETACIÓN DE LOS RE- SULTADOS OBTENIDOS}

En primer Iugar, es destacable que las respuestas encontradas presentan aspectos cualitativamente similares a los que se han señalado en otros trabajos (Sebastiá 1984, Solís 1984, Driver 1986). De una forma muy resumida podemos resaltar lo siguiente:

(A) Es frecuente la asociación entre fuerza y velocidad del movimiento $(76.4 \%)$.

(B) En los movimientos curvilineos existe una tendencia importante a introducir fuerzas compensadoras de carácter centrífugo $(67.2 \%)$.

(C) Parecen detectarse tipos de respuestas bastante generalizados, siendo muy baja la resolución correcta de Ios casos propuestos, completando la secuencia de cuestiones, tanto individualmente (Tabla II) como en grupo (Tabla III).

Tabla II

Frecuencias de respuestas correctas a todas las cuestiones de cada caso propuesto en la resolución individual de la prueba inicial de dinámica

Porecntajc medio de cxitos: $11,9 \%(\mathrm{~N}=67)$.

\begin{tabular}{|c|c|c|}
\hline CASOS PROPURETOS & $\begin{array}{l}\text { PRBCtrancis } \\
\text { ABg. ExITOS }\end{array}$ & $\begin{array}{l}\text { PRBCUERTCIA } \\
\text { RER. EXITTOS }(x)\end{array}$ \\
\hline Honeda & 7 & 10.4 \\
\hline Baion & 6 & 9.0 \\
\hline Luna & \pm 0 & 23.9 \\
\hline Pondulo simple & $\begin{array}{lllll} & \end{array}$ & 20.4 \\
\hline Poncule conico & 9 & 53.4 \\
\hline Honda & 3 & 4. 5 \\
\hline
\end{tabular}


Tabla III

Frecuencias de respuestas correctas a todas las cuestiones de cada caso propucsto en ta resolución en grupo de la prueba inicial de dinámica. Porcentaje medio de éxitos: $18.9 \%(\mathrm{~N}=15)$.

\begin{tabular}{|c|c|c|}
\hline CNSOS PROPUESTOS & $\begin{array}{l}\text { PRECUEaciA } \\
\text { ABS. 8XIOOS }\end{array}$ & $\begin{array}{l}\text { PRBCUETCIA } \\
\text { REL. EXITOS (\%) }\end{array}$ \\
\hline moneda & 0 & 0.0 \\
\hline gasion & 0 & 0.0 \\
\hline Luna & 6 & 40.0 \\
\hline Pondulo simpie & 5 & 33. 3 \\
\hline Psnduse conico & 3 & 20.0 \\
\hline Honda & 3 & 20.0 \\
\hline
\end{tabular}

(D) Las respuestas erróneas, desde el punto de vista del esquema conceptual newioniano, son frecuentes y persistentes después del tratamiento a corto plazo $(83 \%$ antes y $72 \%$ después), aunque menores en los adolescentes formales ( $79 \%$ antes y $55 \%$ después) que en los concretos ( $85 \%$ antes y $85 \%$ después).

En segundo lugar, los resultados que se desprenden deI análisis realizado en el contraste estadístico de las hipótcsis nos permite establecer las siguientes interpretaciones:

$I^{2}$. Los estudiantes con competencia formal o concreta en el razonamiento proporcional dan respuestas alternativas a las del esquema newtoniano que inicialmente son similares. Además, no hemos encontrado nin- guna diferencia estadísticamente significativa entre ambos tipos de sujetos respecto de las puntuaciones alcanzadas en la prueba realizada al principio $(t=1.09$; g.l. $=63 ; p>0.20$ ). Los datos se indican en la Tabla IV.

$2^{a}$. Por el contrario, aparece una diferencia significativa entre las mismas categorias de individuos, después del período de aprendizaje realizado, al comparar sus puntuaciones medias en la prueba final de dinámica. Esta diferencia es a favor de los alumnos con competencia formal $(t=6.35 ; \mathrm{g} .1 .=63 ; \mathrm{p}<0.001 ; \mathrm{rb}$, $p=0.63$ ). En la Tabla $V$ se muestran los datos correspondientes.

$3^{a}$. Después del período de aprendizaje, la evolución de los sujetos formales, aunque no resulta espectacular, es significativamente positiva $(t=4.00 ; \mathrm{g} .1 .=26$; $p<0.001 ; \tau b, p=0.62$ ), correspondiendo los mejores resultados a los que tienen más consolidada su competencia formal. En el mismo período y en las mismas condiciones de aula, los individuos operatoriamente concretos no parecen progresar en el sentido indicado $(\mathrm{t}=0.079 ; \mathrm{g} .1 .=37 ; \mathrm{p}>0.20)$. En la Tabla VI se muestran las frecuencias de estudiantes formales/concretos que varian o conservan sus puntuaciones entre las dos pruebas. La diferencia de puntuaciones se ha considerado estadísticamente significativa si alcanza como minimo el valor 2.7 , calculado con un error máximo del $5 \%$ a partir de los coeficientes de fiabilidad de ambas pruebas.

Tabla IV

Frecuencias de puntuaciones distribuidas por niveles cognitivos en la prucba inicial de dinámica y estadisticos asociados $(N=65)$.

\begin{tabular}{|c|c|c|c|c|c|}
\hline $\begin{array}{l}\text { NIVEIES } \\
\text { PUNTUACI ONES }\end{array}$ & IIA & I I B & I I I A & II & TOTAL \\
\hline $\begin{array}{l}9.3 \\
8.7 \\
7.3 \\
6.7 \\
6.0 \\
4.7 \\
4.0 \\
3.3 \\
2.7 \\
2.0 \\
1.3 \\
0.7 \\
0.0\end{array}$ & $\begin{array}{l}0 \\
0 \\
0 \\
0 \\
0 \\
0 \\
0 \\
0 \\
0 \\
1 \\
1 \\
4 \\
4\end{array}$ & $\begin{array}{l}1 \\
0 \\
1 \\
0 \\
1 \\
2 \\
0 \\
0 \\
2 \\
2 \\
4 \\
6 \\
9\end{array}$ & $\begin{array}{l}0 \\
1 \\
0 \\
0 \\
1 \\
0 \\
1 \\
1 \\
2 \\
0 \\
1 \\
4 \\
1\end{array}$ & $\begin{array}{l}0 \\
0 \\
0 \\
1 \\
0 \\
1 \\
0 \\
0 \\
1 \\
1 \\
6 \\
2 \\
3\end{array}$ & $\begin{array}{l}1 \\
1 \\
1 \\
1 \\
2 \\
3 \\
1 \\
1 \\
5 \\
4 \\
12 \\
18 \\
17\end{array}$ \\
\hline $\begin{array}{l}\operatorname{TOTAL}_{\bar{X}_{i}} \\
\operatorname{Si}_{i} \\
\text { Si }(n-1)\end{array}$ & $\begin{array}{l}10 \\
0.61 \\
0.63 \\
0.66\end{array}$ & $\begin{array}{l}28 \\
1.81 \\
2.39 \\
2.43\end{array}$ & $\begin{array}{l}12 \\
2.63 \\
2.49 \\
2.60\end{array}$ & $\begin{array}{l}15 \\
1.69 \\
1.75 \\
1.83\end{array}$ & $\begin{array}{l}65 \\
1.75 \\
2.17 \\
2.19\end{array}$ \\
\hline $\begin{array}{l}\text { SUJETOS FORHALES: } \\
\text { SUJFTOS COHCRETOS: }\end{array}$ & $\begin{array}{l}N_{F}=27 ; \\
N_{c}=38 ;\end{array}$ & $\begin{array}{l}\bar{x}_{F}=2.10 ; \\
\bar{x}_{c}=1.50 ;\end{array}$ & $\begin{array}{l}S_{F}=2,17 ; \\
S_{C}=2.14\end{array}$ & \multicolumn{2}{|c|}{$S_{F}(n-1)=2 \cdot 21$} \\
\hline
\end{tabular}


Tabla $\mathrm{V}$

Frecuencias de puntuaciones distribuidas por niveles cognitivos en la prueba final de dinámica y estadisticos asociados $(\mathrm{N}=65)$.

\begin{tabular}{|c|c|c|c|c|c|}
\hline $\begin{array}{l}\text { II VELES } \\
\text { PUITuciones }\end{array}$ & $I I A$ & IIB & IIIA & IIIB & Toret. \\
\hline $\begin{array}{l}9.0 \\
9.0 \\
7.0 \\
8.5 \\
5.5 \\
5.0 \\
4.5 \\
4.0 \\
3.0 \\
2.5 \\
1.0 \\
0.0\end{array}$ & $\begin{array}{l}0 \\
0 \\
0 \\
0 \\
0 \\
0 \\
0 \\
0 \\
1 \\
1 \\
0 \\
2\end{array}$ & $\begin{array}{l}0 \\
0 \\
0 \\
0 \\
1 \\
1 \\
1 \\
0 \\
4 \\
5 \\
7 \\
9\end{array}$ & $\begin{array}{l}0 \\
1 \\
0 \\
1 \\
2 \\
0 \\
0 \\
1 \\
3 \\
2 \\
1 \\
1\end{array}$ & $\begin{array}{l}1 \\
1 \\
1 \\
3 \\
1 \\
1 \\
3 \\
1 \\
0 \\
2 \\
1 \\
0\end{array}$ & $\begin{array}{r}1 \\
2 \\
1 \\
4 \\
4 \\
2 \\
4 \\
2 \\
8 \\
10 \\
15 \\
12\end{array}$ \\
\hline $\begin{array}{l}\text { TOTAL } \\
\overline{x_{1}} \\
S_{1} \\
S_{1}(n-1)\end{array}$ & $\begin{array}{l}10 \\
1.15 \\
0.90 \\
0.94\end{array}$ & $\begin{array}{l}20 \\
1.68 \\
1.61 \\
1.64\end{array}$ & $\begin{array}{l}12 \\
3.71 \\
2.20 \\
2.30\end{array}$ & $\begin{array}{l}15 \\
5.17 \\
2.09 \\
2.17\end{array}$ & $\begin{array}{l}\text { 65 } \\
\text { 2. } 77 \\
\text { 2. } 36 \\
\text { 2. } 38\end{array}$ \\
\hline $\begin{array}{l}\text { SUJETOS PORYLLS: } \\
\text { SUJETOS CORCRETOS: }\end{array}$ & $\begin{array}{l}N_{F}: 27 \\
N_{c}=38\end{array}$ & $\begin{array}{l}\overline{\bar{x}}_{F}=4.52 i \\
\vec{x}_{c}=1.53 i\end{array}$ & $\begin{array}{l}s_{5}=2.26 \\
s_{c}=1.47\end{array}$ & $s_{F}(n$ & $=2.31$ \\
\hline
\end{tabular}

Tabla VI

Evolución de las puntuaciones de los sujetos formales/concretos entre las dos pruebas de dinámica.*

\begin{tabular}{|c|c|c|}
\hline $\begin{array}{l}\text { EVoLUCIOH DE } \\
\text { PUATUACTOIFS }\end{array}$ & $\begin{array}{l}\text { PREC. AZURGOS } \\
\text { PORHLLES }\end{array}$ & $\begin{array}{l}\text { PREC. NLUREOS } \\
\text { COICREAOS }\end{array}$ \\
\hline Hejoran & $15(55.6)$ & $\$ 110.51$ \\
\hline No cambian & $11(40.7)$ & 29 (76. 3) \\
\hline Erpeoran & $\pm\{3.7\}$ & $5(13.2)$ \\
\hline
\end{tabular}

* Entre paréntesis se indican las frecuencias relativas, expresadas en tanto por ciento.

\section{CONCLUSIONES E IMPLICACIONES EDUCATIVAS}

Tal y como apuntan los resultados de nuestro trabajo, Ia competencia formal, al menos en el razonamiento proporcional, resulta una condición necesaria para adquirir esquemas conceptuales como el newtoniano. Sin embargo, no parece ser suficiente al no asegurar un éxito importante en la comprensión de los conceptos puestos en juego, a pesar de las especiales caracteristicas de los materiales de aprendizaje utilizados y de la metodología empleada. Así pues, parece claro que una parte de las actividades en el aula deben destinarse a favorecer el desarrollo cognitivo de los escolares; pero esto no basta, porque los estudiantes encuentran generalmente muchas dificultades para aplicar las estrategias adecuadas en la correcta resolución de las tareas cientificas (Carretero 1987 a).

No obstante, no debemos dejarnos invadir por el posible pesimismo de los datos psicológicos, sino más bien tomarlos en consideración para continuar la búsqueda de acciones en el aula más cuidadosamente diseñadas ya que, como acertadamente ha señalado Carrete- ro (1987 b), conviene distinguir ante el adolescente psicológico y el adolescente alumno. Nuestros datos se han obtenido teniendo en cuenta ambos aspectos y aunque no muestren una mejora elevada, ésta resulta apreciable para algunos sujetos. Quizás el método empleado no era todo lo bueno que podría esperarse, o bien, el periodo de aprendizaje fue demasiado corto, o ambas cosas a la vez. Tendremos que esperar un tiempo para ver si la metodologia conduce a resultados distintos a más largo plazo.

¿Qué consecuencias deberiamos obtener entonces para la enseñanza de las ciencias experimentales? Por una parte, es importante disponer de algunos datos sobre la capacidad intelectual de los escolares. Por otra, desde nuestra posición, resulta imprescindible la explicación de las ideas intuitivas o esquemas alternativos de los estudiantes, para lo cual es necesario crear instrumentos adecuados para su detección en el aula. También es precisa la búsqueda de los procedimientos metodológicos más apropiados para superar dichos esquemas conceptuales. Desde esta perspectiva, la corriente constructivista podria resultar muy fecunda en la orientación de los aspectos prácticos de la didáctica de las 
ciencias (Gil 1986). Sin embargo, como advierte oportunamente Pozo (1987 b), las estrategias deberán ser diversas, dada la complejidad que supone el aprendizaje de las ciencias; esto es, en la situacion actual y probablemente en los próximos años, modas aparte, habrá que hacer una utilización holística de los diversos modelos metodológicos y didácticos, tratando de integrar los diferentes aspectos que resulten validados por la investigación en situaciones de aula.

Desde el punto de vista del diseño de materiales para el aprendizaje, nos parece necesario atender a dos aspectos complementarios. En primer lugar, hay que conocer la estructura lógica de la materia a enseñar, es decir, las ideas científicas y su evolución histórica. En segundo Iugar, no podemos descuidar la estructura psicológica en relación con las capacidades cognitivas de los alumnos, las ideas intuitivas o los esquemas conceptuales alternativos de éstos, así como los factores afectivos y los intereses de los escolares, entre otros aspectos destacados (Pozo 1987 a).

Además, puesto que es preciso reconocer, a menos que se adopten posturas inductivistas ingenuas al aplicar el método cientifico (Gil 1983), que los resultados experimentales per se no permiten determinar el conocimiento conceptual necesario para su explicación, los

\section{ANEXO}

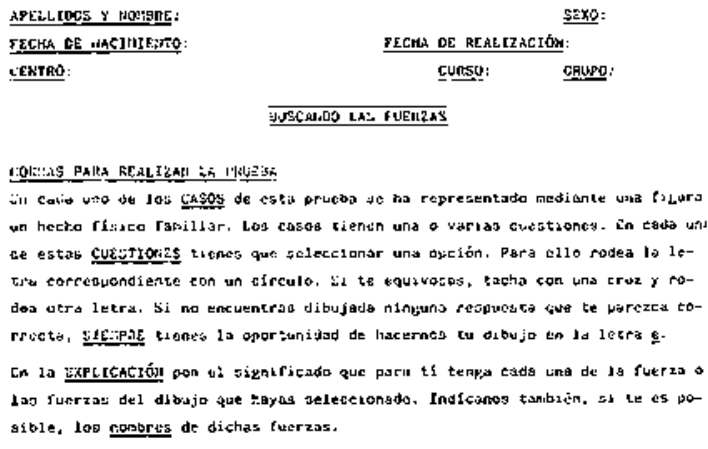

POE PAYOR, POAI SIEKPFE TU EXPLICACIOBi

CR:D 18

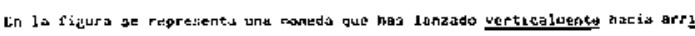

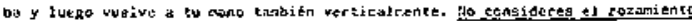
con es atre. Se trate de descubrir lag rucrzap que actúun sobre la bonedic on 10s pasictonela $A, B$ y $C$.

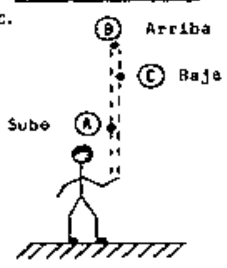

CUEstisa 1 A

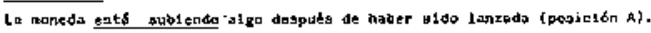

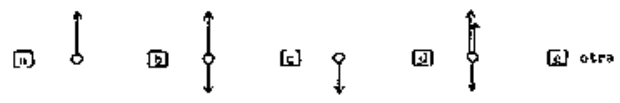

EXPLICACIOH, materiales para el aprendizaje deberán estar enfocados tanto a facilitar la adquisición de los hábitos propios de los procesos de las ciencias, como a favorecer la génesis de los conceptos como construcciones resultantes de la interacción entre los datos experimentales, el marco conceptual del alumno y otros alternativos, propuestos a veces por sus compañeros y otras por el propio profesor. De esta manera estaremos evitanto una imagen estática y acabada de las producciones científicas a la vez que nuestros escolares aprenden y hacen ciencia.

$\mathrm{Si}$ queremos que lo anterior se consiga de un modo efectivo es necesario que los estudiantes dispongan de las competencias cognitivas apropiadas, para lo cual tendrán que haber seguido una escolarización, suficientemente larga, destinada preferentemente, aunque no de modo exclusivo, a potenciar sus capacidades operatorias intelectuales. Luego, en las Enser̃anzas Medias, deben iniciar otra etapa tendente a la superación de sus concepciones de partida desde la óptica de una metodología más rigurosa en sus fines (Gil 1986, 1987). En definitiva, creemos que el aprendizaje y el desarrollo cognitivo de los alumnos vienen a ser como las dos caras de una misma moneda. La escolarización debería ayudar a mejorar ambos aspectos, lo que no parece ser todavia tan frecuente como deseable.

CUES:16: 14

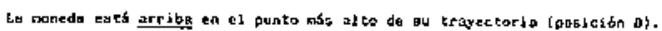

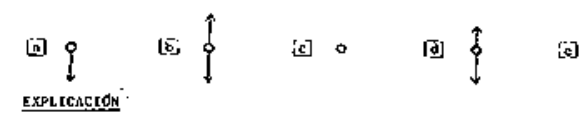

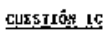

La moneda fate objando (pasicson c).

[aj 8 [is 0 [i]

CASO Z2:

En 1a figura fe representa un bulón que has lanadado en garbboln hactio wita canás

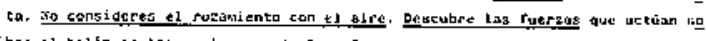
bre el bolion en las poslezont: $A, D, y C$.

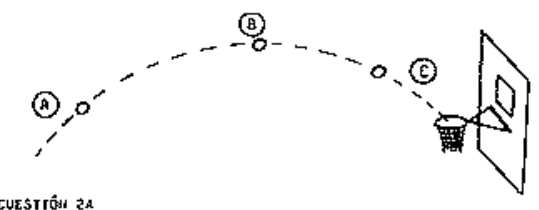

CUESIrớ: 2A

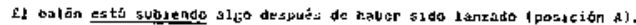

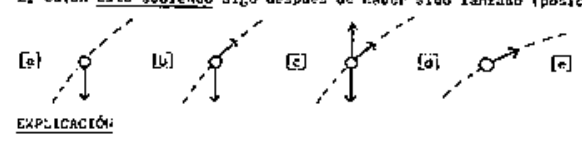

SUEST10́:3 22

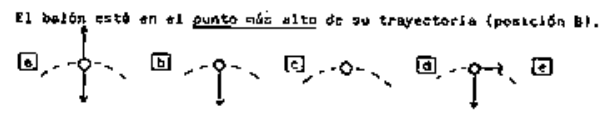

ENSEÑANZA DE LAS CIENCIAS, 1989, 7 (1) 
EXPLICACIŌN

CUESTIOA 32

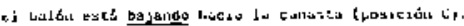

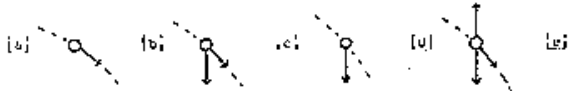

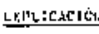

téso 3*

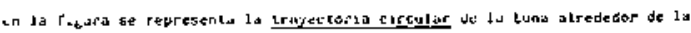

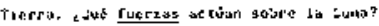

cucsirio:

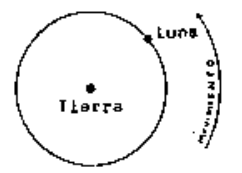

isis

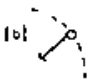

ici

"is is

ExpLICKCIÓN

CASO.as

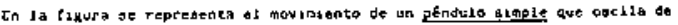

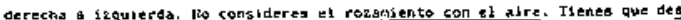
cubrir las fuerzas que setúlan ealiere a bolity en las posictonts A, B y th.

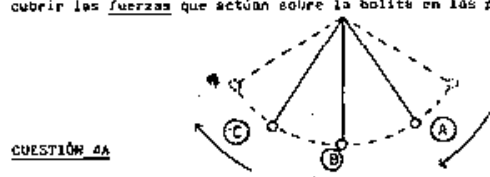

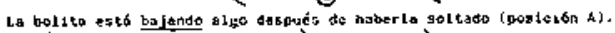

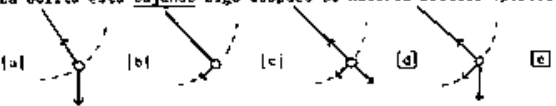

EXPLICACIỎ,

CUESTIÓ: AB

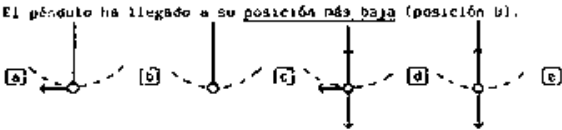

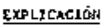

Cuestrón: 4 C

Lo bolito del pendulo asté gubiendo (posiciegn C).

回

(6)<smiles>C[C@@H]1[C@@H](C(C)(C)C)C[C@H]1C</smiles>
เฮ<smiles>CC(C)(C)C</smiles>
回<smiles>COC</smiles>

2xaterectom chso st

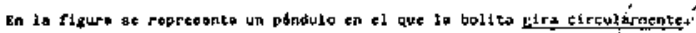

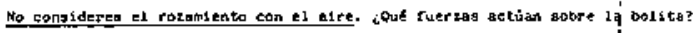

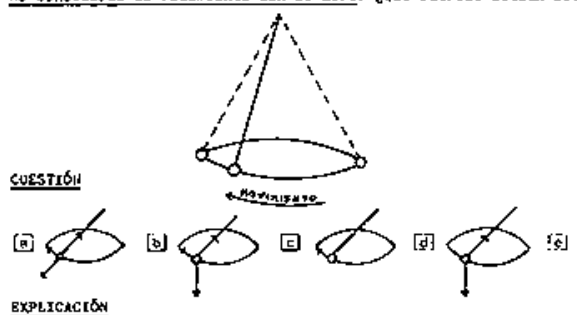

CASOS.60

En 12 Pigura de repreatente el karimiento de und pledra atuda y una everdo quo tu haces ghrar mantensfincole tenag. Alendo la trayectoria circular y pergen-

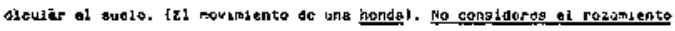
can el aire. Averigut las fuarzas gub actian tobre la piedra en lis posicionso A, B, C Y, D.

(B)

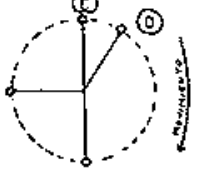

(B)

cUEsTrold Ex

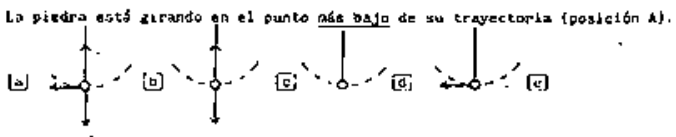
ERLICACIÓ,

CULSTICS; 63

ia piedra está subisndo cono se ind1ed en la gostclón D.

[จ]

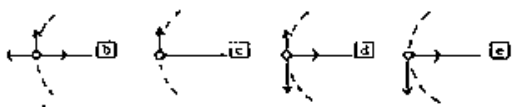

Explicación:

cusstión oc

La pledra sikue dirando y ha alcanzado el punto dis alto de so trayectoris (pousctón $\mathrm{Cl}$.

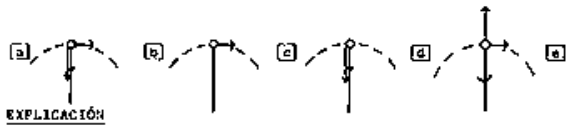

CUESTICH 60

La piedra esté bajanda ceco ze indica en ia pegieition D.

回

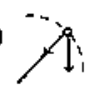<smiles>CC(C)(C)C(C)(C)C</smiles>
(a)<smiles>COC(C)C</smiles>
(6)

\section{REFERENCIAS BIBLIOGRAFICAS}

ACEVEDO, J.A., BOLÍVAR, I.P., SÁNCHEZ-LAULHE, E. y TRUJILLO, M., 1987 a. Razonamiento proporcional lineal de primer grado: la tarea ALTO-CORTO. Aclas de las $V$ Jornadas de Estudio sobre Investigación en la Escuelo, pp. 84-86. (Publicaciones de la Universidad de Sevilia).
ACEVEDO, J.A., BOLÍVAR, J.P., SÁNCHEZ-LAULHE, E. y TRUJILLO, M., 1987 b. Razonamiento proporcional múltiple: Ia tarea ARQUITECTO. Actas de las V Jornadas de Estudio sobre Investigación en la Escuela, pp. $80-83$.

(Publicaciones de la Universidad de Sevilla). 
AGUIRRE DE CÁRCER, I., 1985. Los adolescentes y el aprendizaje de las Ciencias. (Publicaciones de! MEC: Madrid).

CARRASCOSA, J., 1983. Errores conceptuales en la enseñanza de las ciencias: selección bibliográfica. Enseñanza de las Ciencias, Vol. l (1), pp. 63-65.

CARRASCOSA, J., 1985. Errores conceptuales en la ensenanza de la física y quimica: una revisión bibliográfica, Enseñanza de las Ciencias, Vol. 3 (3), pp. 230-234.

CARRETERO, M., 1980. Desarrollo intelectual durante la adolescencia: competencia, actuación y diferencias individuales, Infancia y Aprendizaje, 12, pp. 81-98.

CARRETERO, M., 1987 a. A la búsqueda de la génesis del método cientifico: un estudio sobre la capacidad para eliminar hipótesis, Infancia y Aprendizaje, 38, pp. 53-68.

CARRETERO, M., 1987 b. Desarrollo cognitivo y educación, Cuadernos de Pedagogia, 153, pp. 66-69.

DRIVER, R., 1986. Psicologia cognoscitiva y esquemas conceptuales de los alumnos, Enseñanza de las Ciencias, Vol. 4 (1), pp. 3-15.

FURIO, C.J., 1986. Metodologias utilizadas en la detección de dificultades y esquemas conceptuales en la enseñanza de la química, Enseñanza de las Ciencias, Vol. 4 (1), pp. 73-77.

GIL, D., 1983. Tres paradigmas básicos en la enseñanza de las ciencias, Enseñanza de las Ciencias, Vol. 1 (1), pp. 26-33.

GIL, D., 1986. La metodologia cientifica y la enseñanza de las ciencias: unas relaciones controvertidas, Enseñanza de las Ciencias, Vol. 4 (2), pp. 111-121.

GIL, D., 1987. Los errores conceptuales como origen de un nuevo modelo didáctico: de la búsqueda a la investigación, Investigación en la Escuela, 1, pp. 35-42.

GRUPO RECERCA-FARADAY, 1985. Física: un enfoque conceptual, experimental e histórico. Programas guia para la clase de física. (ICE de la Universitat Autònoma: Barcelona).

HOLTON, G., 1978. The Scientific Imagination: Case Studies, (Cambridge University Press: Cambridge). (Traducción castellana de J.J. Utrilla, 1985. La imaginación cientúfica. Fondo de Cultura Económica: México).
INHELDER, B. y PIAGET, J., 1955. De la logique de l'enfant a la logique de l'adolescent. Essais sur la construction des structures opératoires formalles, (PUF: Paris). (Traducción castellana de M.T. Cevasco, 1972. De la logica del niño a la lógica del adolescente, (Paidós: Buenos Aires).

KEIL, F.C., 1981. Constraints on knowledge and cognitive development, Psychological Review, Vol. 88 (3), pp. 197-227.

OTERO, J. y BRINCONES, I., 1987. El aprendizaje significativo de la segunda ley de la termodinámica, Infancia y Aprendizaje, 38, pp. 89-107.

POZO, J.I. 1987 a. Aprendizaje de la ciencia y pensamiento causal. (Visor: Madrid).

POZO, J.1., 1987 b. Y, sin embargo, se puede enseñar ciencia, Infancia y Aprendizaje, 38, pp. 109-113.

POZO, J.I. y CARRETERO, M., 1987. Del pensamiento formal a las concepciones espontáneas: iqué cambia en la enseñanza de la ciencia? Infancia y Aprendizaje, $38, \mathrm{pp}$. 35-52.

RUTHERFORD, J., 1971. Harvard Projects Physics: An Integrated Science Course. En Tendences nouvelles de l'integration des enseignements scientifiques. (UNESCO: París).

SEBASTIÁ, J.M., 1984. Fuerza y movimiento: la interpretación de los estudiantes, Enseñanza de las Ciencias, Vol. 2 (3), pp. 161-169.

SEBASTIÄ, J.M., 1987 a. Cognitive constraints and spontaneous interpretations in physics, Second International Seminar Misconceptions and Educational Strategies in Sciences and Mathemaics. (Cornell University: Ithaca. New York).

SEBASTIÁ, I.M., 1987 b. ¿Es realmente posible el cambio conceptual? II Congreso Internacional sobre Investigación en la Didáctica de las Ciencias y de las Matemáticas. Valencia. (I.C.E. de la Universidad Autónoma de Barcelona).

SOLÍS, R., 1984. Ideas intuitivas y aprendizaje de las ciencias, Enseñanza de las Ciencias, Vol. 2 (2), pp. 83-89.

VIENNOT, L., 1979 a. Le raisonnement spontané en dynamique élémentaire. (Hermann: Paris).

VIENNOT, L., 1979 b. Spontaneous reasoning in elementary dynamics, European Journal of Science Education, 1, pp. 205-221. 